Cahiers de philosophie de l'université de

\title{
Clausewitz versus Foucault : regards croisés sur la guerre
}

Audrey Hérisson

\section{(2) OpenEdition}

1 Journals

Édition électronique

URL : https://journals.openedition.org/cpuc/300

DOI : 10.4000/cpuc.300

ISSN : 2677-6529

Éditeur

Presses universitaires de Caen

Édition imprimée

Date de publication : 1 novembre 2018

Pagination : 143-162

ISBN : 978-2-84133-904-4

ISSN : 1282-6545

\section{Référence électronique}

Audrey Hérisson, «Clausewitz versus Foucault : regards croisés sur la guerre », Cahiers de philosophie de l'université de Caen [En ligne], 55 | 2018, mis en ligne le 01 novembre 2019, consulté le 31 janvier 2023. URL : http://journals.openedition.org/cpuc/300 ; DOI : https://doi.org/10.4000/cpuc.300

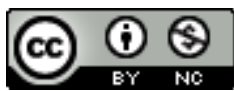

Creative Commons - Attribution - Pas d'Utilisation Commerciale 4.0 International - CC BY-NC 4.0 https://creativecommons.org/licenses/by-nc/4.0/ 


\title{
Clausewitz versus Foucault : regards croisés sur la guerre
}

\begin{abstract}
U'est-ce que la guerre ? Karl von Clausewitz a donné une des réponses certainement les plus connues à cette question. Il écrit dès les premières pages de De la guerre: «[1]a guerre est une simple continuation de la politique par d'autres moyens ${ }^{1}$. Il définit la guerre, en une courte formule, à partir de la politique, en reliant l'une à l'autre dans un rapport de «continuation». Pourtant, Clausewitz avait dégagé un peu avant ce passage de De la guerre une autre acception de la guerre, un concept "pur» de la guerre comme duel.
\end{abstract}

La guerre n'est rien d'autre qu'un duel à une plus vaste échelle. Si nous voulions saisir en une seule conception les innombrables duels particuliers dont elle se compose, nous ferions bien de penser à deux lutteurs. Chacun essaie, au moyen de sa force physique, de soumettre l'autre à sa volonté; son dessein immédiat est d'abattre l'adversaire, afin de le rendre incapable de toute résistance ${ }^{2}$.

Clausewitz repousse cette conception absolue de la guerre, qui entraîne mécaniquement une montée aux extrêmes, pour s'attacher à définir la guerre dans sa réalité, du moins dans celle qu'il observe, c'est-à-dire la guerre interétatique de la fin du XVIII et du début du XIX ${ }^{e}$ siècle en Europe. Il conçoit, à partir de là, la guerre comme un véritable instrument de la politique, cette dernière démontrant des capacités à mettre la violence sous contrôle, à maîtriser l'action engagée dans les luttes, en bref, à domestiquer la guerre. La formule selon laquelle la guerre est la continuation de la politique par d'autres moyens est certainement une des plus commentées de la pensée clausewitzienne et a fait l'objet de nombreuses interprétations.

1. K. von Clausewitz, De la guerre [1832], D. Naville (trad.), Paris, Minuit, 1955, p. 67.

2. Ibid., p. 51. 
Plusieurs auteurs ont voulu retourner cette formule: Lénine, Ludendorff, Carl Schmitt, Michel Foucault, René Girard ${ }^{3}$, entre autres.

Le pouvoir, c'est la guerre, c'est la guerre continuée par d'autres moyens. Et à ce moment-là, on retournerait la proposition de Clausewitz et on dirait que la politique, c'est la guerre continuée par d'autres moyens ${ }^{4}$.

Foucault renverse la formule de Clausewitz en 1976 dans son cours au Collège de France, au moment même où Aron ${ }^{5}$ réintroduit l'œuvre de Clausewitz en France, et où Michael Howard et Peter Paret ${ }^{6}$ offrent une nouvelle traduction en langue anglaise de Vom Kriege. Cette année-là, Aron, Howard et Paret, que nous pouvons qualifier de penseurs libéraux, ancraient durablement la formule de Clausewitz, non renversée, dans la pensée politique et stratégique occidentale. Le contexte politico-militaire du milieu des années 1970 est particulièrement chargé par les conflits internationaux mais aussi par les luttes sociales en France après 19687. Le cours de 1976 au Collège de France, Il faut défendre la société, est essentiellement consacré à l'analyse du pouvoir dans ses liens avec la guerre.

Le pouvoir, tout simplement, est-il une guerre continuée par d'autres moyens que les armes ou les batailles ${ }^{8}$ ?

Foucault utilise la guerre comme principe historique de fonctionnement du pouvoir politique et tente de repérer les rapports de force à l'intérieur de la société. Le renversement de la formule clausewitzienne se trouve au centre de ses réflexions sur l'histoire des luttes politiques et du pouvoir. Clausewitz essayait de dégager une caractérisation des guerres réelles qu'il observait, c'est-à-dire des guerres interétatiques. L'utilisation de sa célèbre formule, sortie de la ligne argumentative dans laquelle elle s'insérait, a souvent eu tendance à donner un caractère universel à une réflexion qui n'opérait - comme celle de Foucault - que dans un contexte géographique, historique et culturel particulier.

3. A. Hérisson, "La "montée aux extrêmes" chez René Girard: une lecture du concept clausewitzien de la guerre", Les cahiers de la Revue Défense Nationale, 2015, en ligne à l'adresse suivante: https://fr.calameo.com/read/ooo558115da5e3094f35e.

4. M. Foucault, Il faut défendre la société. Cours au Collège de France, 1975-1976, Paris, Gallimard - Seuil (Hautes études), 1997, p. 15-16.

5. R. Aron, Penser la guerre, Clausewitz, Paris, Gallimard (Bibliothèque des sciences humaines), 1976, t. I, L'âge européen. Aron désigne par le simple terme de «formule» la citation célèbre de Clausewitz.

6. K. von Clausewitz, On War, M. Howard, P. Paret (éd. et trad.), Princeton, Princeton University Press, 1976.

7. A. Fontana, "Situation du cours», in Lectures de Michel Foucault, J.-C. Zancarini (dir.), Paris, ENS Éditions, vol. 1, À propos de "Il faut défendre la société", 2001, p. 37-58.

8. M. Foucault, Il faut défendre..., p. 18. 
Dans la perspective d'une recherche de définition «universelle», les questions "qu'est-ce que la guerre?» et «qu'est-ce que la politique?" se répondent et se renversent l'une dans l'autre dans une circularité - comme le montrent la formule de Clausewitz et ses renversements - dont il semble difficile de sortir. Foucault nous invite à ne plus nous poser ces questions, mais plutôt à poser celle-ci: comment s'exerce la politique ou la guerre? En se focalisant sur le "comment», Foucault nous ramène à une analyse contextualisée du phénomène de la guerre. Il propose ainsi une façon d'aborder la question du rapport entre guerre et politique qui lui permet d'explorer une nouvelle analytique du pouvoir dans laquelle la guerre est l'instrument d'analyse.

Cet article a pour double objectif de montrer comment Foucault utilise les références à l'œuvre de Clausewitz dans sa pensée des rapports entre le pouvoir, la guerre et la politique, puis de relire quelques pages de De la guerre à l'aune de la philosophie foucaldienne pour en dégager des éléments intéressants - résultats de cette perspective croisée - pour la pensée de la guerre.

\section{Les références à la pensée clausewitzienne dans l'analytique foucaldienne du pouvoir}

La formule de Clausewitz de la guerre comme continuation de la politique par d'autres moyens apparaît chez Michel Foucault en 1976, dans le cours au collège de France Il faut défendre la société et dans l'ouvrage La volonté de savoir, mais également en 1978 dans le cours Sécurité, territoire, population. Cependant, ces deux années-là, il n'en fait pas la même utilisation: la première fois, il s'en sert pour traiter la question des rapports entre guerre et politique, la seconde pour traiter celle de la raison politique; la première fois, la formule sert à marquer une rupture - la fin de l'ère des discours historico-politiques -, la seconde, elle sert à marquer une continuité - la persistance de la raison diplomatique depuis les traités de Westphalie. Or, cette dernière continuité sera elle-même rompue dès les années 1830, année de la mort de Clausewitz, de sorte que celui-ci apparaît au cœur de deux tournants majeurs de la rationalité politique en Europe.

Clausewitz ne pense pas la politique pour elle-même et s'arrête à la question des rapports qu'elle entretient avec la guerre. Pourtant, ses écrits feront l'objet d'interprétations à portée politique ${ }^{9}$, notamment par Aron.

9. B. Durieux, Clausewitz en France. Deux siècles de réflexion sur la guerre: 1807-2007, Paris, Economica, 2008. 


\section{Les discours historico-politiques et la formule de Clausewitz}

$\mathrm{Au}$ XIX ${ }^{\mathrm{e}}$ siècle, Clausewitz est témoin d'une transformation, ou plutôt d'une disparition: celle du discours historico-politique. Dans le cours du 7 janvier 1976, Foucault commence par opposer deux formes d'analyse du pouvoir: une analyse classique en termes de contrat, cession et aliénation - celle des philosophes théoriciens du contrat social -, dans laquelle le mécanisme du pouvoir repose essentiellement sur l'oppression; et une analyse non économique du pouvoir, dans laquelle «le pouvoir, c'est la guerre, c'est la guerre continuée par d'autres moyens $»^{10}$. Foucault retourne la formule de Clausewitz et oppose deux grands systèmes d'analyse du pouvoir: le schéma "pouvoir-contrat» et le schéma "guerre-répression». La répression se distingue, chez Foucault, de l'oppression qui est l'abus dans lequel est susceptible de tomber le pouvoir politique mis en place par contrat social. La répression est une action permanente, utilisant contraintes et violences, sur une personne ou un groupe, afin d'empêcher le développement d'un désordre. Dans le premier schéma, l'opposition pertinente est celle du légitime et de l'illégitime, et l'oppression est ponctuelle; dans le second, l'opposition est entre lutte et soumission, et la répression est permanente. Mais pour Foucault, la notion de «répression " est insuffisante pour caractériser les mécanismes et effets de pouvoir.

La répression n'offre qu'un schéma en sens unique du pouvoir, celui du dominant vers le dominé. Or, le cas de la domination pure est extrêmement rare - c'est celui de l'esclavage par exemple - et il faut, pour Foucault, pouvoir penser les rapports de force entre le dominant, au sens faible ${ }^{11}$, et le dominé, au sens faible également puisqu'il résiste. Il lui faut un schéma binaire capable de penser la réciprocité de la lutte. Il a besoin d'un instrument d'analyse basé sur la logique du duel: la guerre telle que la conceptualise Clausewitz paraît être le candidat idéal pour l'analytique foucaldienne du pouvoir. Chez Foucault, le pouvoir politique produit des discours de vérité qui sont eux-mêmes des techniques et tactiques de domination. Les procédés de domination sont les mêmes que l'on se place dans le cadre d'une domination pure ou dans celui d'une domination au sens faible, c'est-à-dire dans celui où la figure du résistant remplace celle de l'assujetti. Dans ce dernier cas, l'action du pouvoir politique produit également, en réaction, des discours de vérité qui sont instruments de révolte et que Foucault qualifie d'historico-politiques.

10. M. Foucault, Il faut défendre..., p. 16-17.

11. La notion de domination «au sens faible» n'est pas de Foucault. Nous l'introduisons ici pour la distinguer de la notion de domination employée dans un sens plus absolu et éviter ainsi toute confusion. 
Ces discours historico-politiques sont pour Foucault les discours d'une «guerre des races ${ }^{12}$, d'une "guerre qui travaille la société et la divise sur un mode binaire»; ils sont "comme [la] matrice de toutes les formes sous lesquelles, ensuite, on recherchera le visage et les mécanismes de la guerre sociale ${ }^{13}$. Or, à partir de la Révolution, cet élément de la guerre, constitutif de l'intelligibilité historique au XVIII ${ }^{\mathrm{e}}$ siècle, va être en quelque sorte apprivoisé ${ }^{14}$. La théorie de la guerre des races subit en effet deux transcriptions, celle d'une théorie des races au sens historico-biologique s'articulant sur les mouvements des nationalités en Europe ou sur la politique de la colonisation européenne, et celle de la théorie de la guerre sociale se développant au début du XIX ${ }^{e}$ siècle pour se définir comme lutte de classes. Cette distinction entre guerre des races et lutte des classes est importante car Marx vise à long terme la suppression de la division sociale et l'avènement d'une classe unique; il aspire donc à la disparition de ce qui sous-tend le discours historico-politique, c'est-à-dire la division binaire. Mais la première transcription, qui est une construction plus historico-biologique qu'historico-politique, celle qui reprend le thème de l'affrontement des races dans la théorie de l'évolutionnisme et de la lutte pour la vie, tend également à faire disparaître le modèle binaire de l'affrontement au sein de la société.

[C]e que nous voyons [apparaître] comme polarité, comme cassure binaire dans la société, ce n'est pas l'affrontement de deux races extérieures l'une à l'autre; c'est le dédoublement d'une seule et même race en une sur-race et une sous-race. Ou encore: la réapparition, à partir d'une race, de son propre passé. Bref, l'envers et l'en-dessous de la race qui apparaît en elle ${ }^{15}$.

Ce qui intéresse Foucault, dans Il faut défendre la société, c'est cette première transcription. Alors que la lutte des classes oppose clairement le prolétariat - issu de la partie pauvre du Tiers-État - à la bourgeoisie, cette transcription historico-biologique ne désigne plus les lutteurs. Foucault explique cette

12. M. Foucault, Il faut défendre..., p. 67. Foucault n'utilise pas le terme de race dans le sens commun; il le définit ainsi: «[...] dans ce discours on dit, qu'il y a deux races lorsqu'on fait l'histoire de deux groupes qui n'ont pas la même origine locale; deux groupes qui n'ont pas, du moins à l'origine, la même langue et souvent pas la même religion; deux groupes qui n'ont formé une unité et un ensemble politique qu'au prix de guerres, d'invasions, de conquêtes, de batailles, de victoires et de défaites, bref de violence; un lien qui n'est établi qu'à travers la violence de la guerre. Enfin, on dira qu'il y a deux races lorsqu'il y a deux groupes qui, malgré leur cohabitation, ne se sont pas mélangés à cause de différences, de dissymétries, de barrages qui sont dus aux privilèges, aux coutumes et aux droits, à la répartition des fortunes et au mode d'exercice du pouvoir ».

13. Ibid., p. 52.

14. Ibid., p. 193-194.

15. Ibid., p. 52. 
transformation par une dialectisation interne, une autodialectisation du discours historique qui correspond à son embourgeoisement. Le rapport de guerre va apparaître avec un rôle négatif: «un rôle non plus constitutif de l'histoire mais protecteur et conservateur de la société» ${ }^{16}$. La guerre n'est plus la condition d'existence des rapports politiques et donc de la société; la guerre est la condition de la survie de la société elle-même. La guerre interne apparaît comme la défense de la société contre les dangers qui naissent au sein d'elle-même. Pour Foucault, c'est en prenant à son compte les discours sur la guerre que la bourgeoisie de la seconde moitié du XVIII ${ }^{e}$ siècle a dialectisé et a fait perdre la trace de la rationalité qui s'en dégageait, celle de l'historicisme. Les théories du «droit naturel », notamment celle du contrat social, ont servi de base à l'effacement d'une histoire sociale.

Au début du XIX ${ }^{\mathrm{e}}$ siècle, lorsque Clausewitz écrit De la guerre, il entérine, selon Foucault, l'effacement des dernières traces de cette guerre sociale historico-biologique - qui deviendra une guerre cachée, insidieuse - au profit de la seule guerre qui demeure visible ${ }^{17}$ : la «grande guerre» entre États. Clausewitz retournerait finalement une formule qui le précédait d'au moins deux siècles: «le principe selon lequel la politique, c'est la guerre continuée par d'autres moyens était un principe bien antérieur à Clausewitz, qui a simplement retourné une sorte de thèse à la fois diffuse et précise qui circulait depuis le XVII ${ }^{e}$ et le XVIII ${ }^{\mathrm{e}}$ siècle ${ }^{18}$.

\section{La raison d'État et le rapport guerre-politique}

Foucault utilise de nouveau la formule de Clausewitz dans le cours suivant au Collège de France, Sécurité, territoire, population, cette fois-ci pour montrer la continuité de la raison politique qui est apparue avec les traités de Westphalie et qu'il nomme "raison d'État». Au Moyen Âge, la guerre était un règlement judiciaire de différends entre détenteurs, ou prétendants à la détention, de pouvoir, ou plutôt de "droits ${ }^{19}$. Avec la raison d'État, la guerre perd sa continuité avec le droit et récupère une continuité avec la politique qui a pour fonction de maintenir l'équilibre entre les États. La politique dit à qui faire la guerre, comment, pour que l'équilibre ne soit pas compromis, et avec qui former alliance.

16. M. Foucault, Il faut défendre..., p. 193-194.

17. L'autre branche de la guerre des races, celle de la lutte des classes, refera surface quelques années plus tard au milieu du XIX ${ }^{\mathrm{e}}$ siècle.

18. M. Foucault, Il faut défendre..., p. 41.

19. M. Foucault, Sécurité, territoire, population. Cours au Collège de France, 1977-1978, Paris, Gallimard - Seuil (Hautes études), 2004, p. 308-309. 
[C]'est à partir de ce moment-là qu'apparaît le principe dont vous savez bien qu'il sera formulé presque deux cents ans après par quelqu'un qui aura dit: «La guerre, c'est la politique continuée par d'autres moyens ». Mais il ne faisait rien d'autre que constater une mutation qui avait été acquise en fait dès le début du XVII ${ }^{\mathrm{e}}$ siècle, [avec la constitution] de la nouvelle raison diplomatique, de la nouvelle raison politique au moment du traité de Westphalie ${ }^{20}$.

Pour Foucault, Clausewitz se conforme à nouveau à la rationalité dominante: après celle de la bourgeoisie qui a su effacer toute trace de la guerre à l'intérieur de la société, il suit celle de la politique extérieure des États européens. Or, le congrès de Vienne qui a réaffirmé en 1815 la raison diplomatique de l'équilibre des rapports de force sera comme le dernier sursis de cet ordre européen bien réglé. Napoléon l'a bien ébranlé, mais les forces en œuvre viendront du fond des sociétés. Néanmoins, au début du XIX ${ }^{\mathrm{e}}$ siècle, lorsque Clausewitz pose le problème des rapports entre politique et stratégie, le congrès de Vienne vient de manifester l'importance politique « des rapports de force et du calcul des rapports de force comme principe d'intelligibilité et de rationalisation des relations internationales $»^{21}$.

Foucault invente le terme de "gouvernementalité » pour analyser le rapport entre les relations de pouvoir et la constitution de l'État en tant que forme de gouvernement ${ }^{22}$. La gouvernementalisation de l'État suppose la mise en place d'un dispositif diplomatico-militaire ayant pour fonction d'assurer le maintien du rapport de force avec ses voisins. Permis par les traités de Westphalie, ce dispositif repose sur trois instruments: la guerre, la diplomatie et un dispositif militaire permanent. L'homme de guerre est professionnalisé; une structure permanente est créée pour le recrutement de l'armée; les territoires s'équipent de forteresses et de réseaux de transport; et enfin se consolident un savoir, une réflexion tactique, des types de manœuvre, des schémas d'attaque et de défense, c'est-à-dire toute une réflexion sur la chose militaire et les guerres possibles.

Là encore, on voit comment ce principe de Clausewitz que la guerre, c'est la politique continuée, a eu un support, un support institutionnel précis qui a été l'institutionnalisation du militaire. La guerre, ce n'est plus une autre face de l'activité des hommes. La guerre, ça va être, à un moment donné, la mise en œuvre d'un certain nombre de moyens que la politique a définis et dont le militaire est une des dimensions fondamentales et constitutives ${ }^{23}$.

\footnotetext{
20. Ibid., p. 308-309.

21. Ibid., p. 93 .

22. Ibid., p. 111.

23. Ibid., p. 313 .
} 
Foucault parle de «complexe politico-militaire» pour qualifier ce mécanisme de sécurité de l'équilibre européen; ce complexe sera mis en jeu en permanence et la guerre ne sera que l'une de ses fonctions. À partir de ce rapport intime entre la paix et la guerre, Clausewitz théorise le rapport du civil et du militaire, rapport qui perdure encore aujourd'hui dans tous les pays occidentaux. Nous pouvons dire que quelque chose de la logique de la «balance de l'Europe» a donc survécu aux aspirations nationales qui ont embrasé l'Europe dès les années 1830. Les réflexions de Clausewitz sur les rapports politico-militaires ont acquis une dimension d'universalité qu'il serait intéressant d'interroger. En effet, la «raison d'État», chez Foucault, se caractérise par une limitation externe de l'exercice du pouvoir des États, notamment par le biais de la balance européenne, et par une illimitation interne de cet exercice du pouvoir sur les populations administrées par l'État. À l'inverse, le basculement, au tournant du XVIII ${ }^{\mathrm{e}}$ siècle, dans une autre raison politique - que Foucault appelle «biopolitique» - s'accompagne d'une autolimitation interne du pouvoir des États - dans un souci, qui se veut bienveillant, de la vie des populations - et d'une illimitation externe de l'exercice de leur pouvoir dans leur recherche de l'accès au marché mondial.

Le problème du traité de Vienne, c'était, bien entendu, de refermer en quelque sorte cette illimitation impériale. C'était, bien entendu, de rétablir l'équilibre européen, mais, au fond, avec deux objectifs différents. [...] c'était de reconstituer un équilibre européen dans l'ancienne forme, celle des XVII et XVIII ${ }^{e}$ siècles $[\ldots]^{24}$.

L'ancienne forme de l'équilibre européen ne perdurera pas et sera vite remplacée par la rationalité politique de la course économique mondiale, donc d'une forme de guerre commerciale illimitée. Quel sens pouvait donc garder la formule de Clausewitz, qui pose la guerre comme instrument maîtrisé par la politique, dans ce contexte de guerre illimitée? Le sens qui a été conservé est celui que souhaitait lui donner le courant idéologique qui promeut, selon Foucault, la biopolitique: le libéralisme. Pour s'adonner sans limite à la guerre économique mondiale, celui-ci a distingué la guerre entre États avec des moyens militaires, celle qui devait rester maîtrisée, de la guerre entre États avec des moyens économiques et financiers.

Des deux utilisations de la formule clausewitzienne par Foucault, nous pouvons retenir le caractère fondamentalement contextuel et mouvant du rapport entre guerre et politique. D'une part, ce rapport suit cette formule, mais inversée, du XVII ${ }^{\mathrm{e}}$ au tournant du XVIII ${ }^{\mathrm{e}}$ siècle, depuis l'avènement

24. M. Foucault, Naissance de la biopolitique. Cours au Collège de France, 1978-1979, Paris, Gallimard - Seuil (Hautes études), 2004, p. 61-62. 
des discours historico-politiques jusqu'à leur dialectisation au moment de la Révolution française, puis s'accorde sur cette même formule non inversée; ces renversements interviennent dans ce que nous pourrions appeler un contexte de politique et de guerre intérieures. D'autre part, le rapport entre guerre et politique, cette fois-ci dans un contexte de relations internationales, suit la formule de Clausewitz - dans le sens où la guerre était maîtrisée par le politique - depuis les traités de Westphalie jusqu'aux années 1830, puis se renverse quasi totalement - avec l'économie politique et la guerre commerciale illimitée -, laissant au champ diplomatico-militaire les vestiges d'une maîtrise politique de la guerre. Dans les deux cas - la distinction «intérieur» ou "extérieur» n'étant d'ailleurs pas totalement satisfaisante -, le renversement ou non de la formule n'est pas net: la dialectisation fait disparaître une guerre des races qui perdure finalement sous les apparences; de même, la guerre maîtrisée politiquement et juridiquement dans le champ des relations interétatiques cohabite avec une guerre débridée dans le champ économique.

Guerre et politique sont intimement liées dans un rapport complexe de renvois. Si Foucault n'a pas souhaité, dans le cadre de ses travaux, entrer dans une conceptualisation de ces notions mais rester dans une analytique, Clausewitz s'est engagé dans les deux démarches. Employées en alternance, celles-ci peuvent s'enrichir l'une de l'autre pour démêler la complexité du lien "guerre-politique» et peut-être parvenir à mieux définir chacun de ces deux objets.

\section{Retour sur l'œuvre de Clausewitz: conceptualisation et analytique de la guerre}

La rédaction de De la guerre fait l'objet d'une polémique qui se cristallise autour du statut de la "guerre absolue». La reconstitution de cette rédaction par Aron ${ }^{25}$ tente de montrer les hésitations, les réécritures, inachevées parfois, des différents livres constituant l'ouvrage. Howard et Paret ${ }^{26}$ aboutissent aux mêmes conclusions qu'Aron: ce n'est qu'à la fin de sa vie que Clausewitz aurait compris le rôle plus qu'essentiel de la politique, à laquelle la guerre est subordonnée; la guerre absolue, qui est une guerre illimitée, est de ce fait irréelle, contrairement à ce que l'on lit parfois dans le traité. Claude Lefort ${ }^{27}$ est très critique quant à la reconstruction d'Aron

25. R. Aron, Penser la guerre...

26. K. von Clausewitz, On War.

27. C. Lefort, «Lectures de la guerre: le Clausewitz de Raymond Aron», Annales. Économies, Sociétés, Civilisations, $32^{\mathrm{e}}$ année, $\mathrm{n}^{\mathrm{o}}$ 6, 1977, p. 1268-1279. 
qu'il compare à un roman policier. Hew Strachan ${ }^{28}$ s'oppose encore plus à cette thèse et estime que la rédaction de l'ouvrage est bien plus achevée que les trois auteurs libéraux ne l'ont écrit en 1976. Il les accuse d'avoir fait de Clausewitz un libéral avant l'heure et d'avoir cherché à étouffer et discréditer sa pensée de la guerre absolue. Nous nous attacherons, dans les analyses qui suivent, à rester au plus près du texte, tel que l'a lu Foucault dans sa traduction française de 1955.

\section{Guerre absolue et guerre réelle}

Commençons par le chapitre 2 du livre VIII qui s'intitule "Guerre absolue et guerre réelle». Nous y trouvons que: «la guerre [est] quelque chose de tout différent de ce qu'elle devrait être d'après son concept - une affaire mitigée, une essence sans cohésion interne. C'est ainsi qu'elle se présente à peu près partout, et l'on pourrait douter de la réalité de notre notion de son essence absolue si nous n'avions pas vu de nos jours la guerre réelle dans sa perfection absolue ${ }^{29}$. Nous avons rappelé en introduction que le concept pur de la guerre selon Clausewitz repose sur le duel et la réciprocité qui montent mécaniquement aux extrêmes. Ce concept fait l'objet de déductions logiques avant d'être "abandonné définitivement", selon Aron, pour la définition trinitaire de la guerre, à la fin du chapitre 1 du livre $\mathrm{I}^{30}$. Dans cette définition, nous retrouvons la nature subordonnée d'instrument politique de la guerre aux côtés du jeu de hasard et de la violence originelle, chacun de ces trois éléments étant rapporté respectivement à «l'entendement pur», «une libre activité de l'âme» et «une impulsion naturelle», et chacun étant également traduit dans le contexte politique de l'époque dans, respectivement, le «gouvernement " ${ }^{31}$, le «commandant et son armée » et le "peuple». Dans le livre VIII néanmoins, il semble que Clausewitz conserve un doute: le concept de guerre, cette essence absolue, pourrait se vérifier dans la réalité. En effet, il a été témoin des guerres napoléoniennes.

Après la courte introduction de la Révolution française, l’impitoyable Bonaparte l'a vite poussée jusqu'à ce point [la guerre réelle dans sa perfection absolue] [...].

28. H. Strachan, Clausewitz's "On War". A Biography, New York, Grove Press, 2007; H. Strachan, The Direction of War. Contemporary Strategy in Historical Perspective, Cambridge, Cambridge University Press, 2013, "The Case for Clausewitz: Reading On War Today», p. 46-63.

29. K. von Clausewitz, De la guerre, livre VIII, p. 673.

30. Ibid., livre I, p. 69.

31. Il est à noter qu'il ne faut pas confondre la notion de gouvernement telle que la conçoit Foucault et le gouvernement comme institution dirigeante tel qu'il est question dans cette citation de Clausewitz. 
N'est-il pas naturel et nécessaire que ce phénomène nous ait ramené au concept originel de la guerre avec toutes ses déductions rigoureuses ${ }^{32}$ ?

Clausewitz écrit revenir sur une réflexion qu'il n'avait jusque-là pas poursuivie. Ceci confirme l'idée d'abandon du concept pur dans le livre I, mais pas son caractère définitif dans tout le traité ${ }^{33}$. La clarté que permet le travail dans l'hypothèse du concept pur est séduisante lorsque l'on veut faire une théorie de la guerre, mais Clausewitz refuse cette facilité intellectuelle, qu'il reproche d'ailleurs aux auteurs qui ont voulu faire une science de la guerre. Clausewitz conclut, à ce niveau de la réflexion ${ }^{34}$, qu'il ne faut pas abandonner la réalité des guerres pour le concept pur et surtout ne pas construire une théorie seulement hypothético-déductive. Mais cela ne veut pas dire que le concept est inutile et n'a plus lieu d'être dans ce traité; surtout, cela ne signifie pas que la réalité des guerres napoléoniennes n'est plus à prendre en compte. Clausewitz construit une analytique de la guerre qui s'intéresse à toutes les expériences de guerre. Il l'explique dans sa préface. Clausewitz ne souhaite délaisser aucune approche au profit d'une autre. «L'investigation et l'observation, la philosophie et l'expérience ne doivent jamais se mépriser ni s'exclure mutuellement; elles sont garantes l'une de l'autre. Les propositions du présent ouvrage et l'architecture serrée de leur nécessité interne reposent sur l'expérience, ou sur le concept de guerre lui-même comme point de repère extérieur, de sorte qu'elles ne sont pas sans fondement» 35 . Le concept pur de guerre est essentiel pour cette analytique; il est bien un repère extérieur à la réalité - ce n'est en effet qu'un concept - mais sert de guide, de cadre à l'analyse de l'expérience.

Ainsi, nous pouvons proposer la lecture suivante. La notion de "guerre absolue» permet à Clausewitz de projeter sur la réalité le concept pur de la guerre, afin de pouvoir comparer des choses comparables: les guerres qu'il appelle «réelles», c'est-à-dire les guerres depuis Alexandre jusqu'à Bonaparte, et la guerre absolue, qui traduit dans la réalité les principes déduits du concept. Jusqu'à Napoléon, les guerres étaient fort éloignées de cette reconstitution fictive, du fait d'un certain nombre de facteurs qu'il énumère patiemment dans son traité. Puis le «Dieu de la guerre»

32. K. von Clausewitz, De la guerre, livre VIII, p. 673.

33. Aron s'appuie sur une note non datée de l'auteur qui y déclare que seul le livre I est dans un état d'achèvement satisfaisant pour montrer qu'il ne faut pas se fier aux autres livres; Clausewitz aurait eu l'intention de les reprendre afin de confirmer l'abandon définitif de la conception absolue de la guerre et d'en retirer tous les doutes et retours au concept, mais n'aurait pas eu le temps de le faire. Strachan estime que Clausewitz a pu poursuivre ses travaux après cette note et surtout qu'il est impossible de savoir ce qu'il aurait modifié ou pas.

34. K. von Clausewitz, De la guerre, livre VIII, p. 673.

35. Ibid., préface, p. 47. 


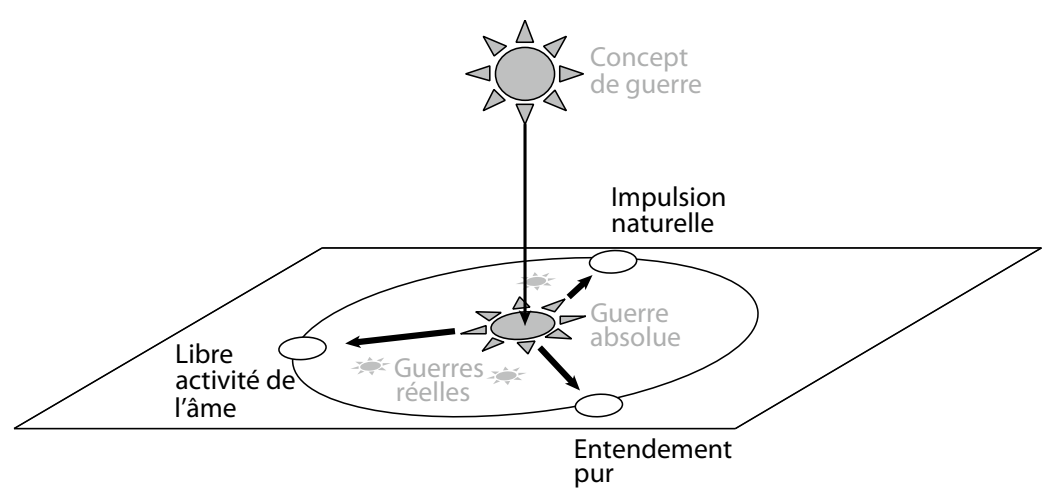

Fig. 1: Schéma représentant les positions relatives entre le concept pur,

la guerre absolue, les guerres réelles, et les trois pôles d'attraction que sont l'impulsion naturelle, la libre activité de l'âme et l'entendement pur, dans la théorie clausewitzienne

a permis d'incarner cette réalité-fiction et, par là, de valider la démarche intellectuelle de Clausewitz. Il n'en reste pas moins que la guerre absolue, réalisée dans les guerres napoléoniennes, est un cas limite, un cas extrême, qui n'est pas amené à se reproduire de façon systématique dans le futur. Nous proposons dans la figure 1 ci-dessus une schématisation des rapports entre les trois différentes notions qui se rapportent à la guerre: le concept, la projection fictive dans la réalité et les phénomènes réels.

Le schéma montre également les trois pôles d'attraction qui modifient l'exercice des guerres réelles et les dévient, en quelque sorte, de la réciprocité pure et absolue du duel. Ce qui intéresse Clausewitz, c'est de décrire comment la guerre s'exerce dans la réalité. À l'instar de Foucault qui fait une analytique du pouvoir, Clausewitz a fait, avant lui, une analytique de la guerre.

\section{Science de la guerre et art de la guerre}

Clausewitz décrit dans le livre II la théorie qu'il entend mettre en place. Il fait la distinction entre la science et l'art de la guerre en utilisant la distinction savoir / pouvoir - notions qui se révéleront clés plus tard dans la philosophie de Foucault. Il faudrait, d'après Clausewitz, utiliser le terme de «science, lorsqu'il s'agit d'aboutir à un savoir» et le terme d' «art, lorsque l'objectif consiste à pouvoir ${ }^{36}$. Or, nous l'avons vu, pour lui, une théorie de la guerre qui est une

36. En allemand, Kunst [art] a la même racine que Können [pouvoir]. 
science n'est d'aucune utilité: elle se cantonne au savoir encyclopédique. Mais un art, seul, ne conviendrait pas non plus. Il semblerait qu'il faille combiner les deux, construire une théorie qui soit un savoir-pouvoir ou un pouvoir-savoir. La lecture de Clausewitz nous ramène à celle de Foucault, à ses dispositifs de savoir-pouvoir que sont notamment les disciplines, et à ses dispositifs de pouvoir-savoir qui apparaissent avec le tournant biopolitique. Ces deux sortes de dispositifs s'assemblent de façon orthogonale pour devenir les instruments du pouvoir normalisateur de l'existence sociale: le bio-pouvoir.

Il vaudrait mieux la [la guerre] comparer, plutôt qu'à un art quelconque, au commerce, qui est aussi un conflit d'intérêts et d'activités humaines; elle ressemble encore plus à la politique, qui peut être considérée à son tour, du moins en partie, comme une sorte de commerce sur une grande échelle ${ }^{37}$.

De même, la comparaison, par Clausewitz, de la guerre avec les conflits d'intérêts économiques et politiques, dont il a été question ci-avant, nous ouvre la voie pour faire un parallèle entre l'économie de guerre que peut mettre en œuvre un État pour assumer le risque de guerre et l'économie politique qui est, chez Foucault, la forme majeure du savoir mis en place par l'État gouvernementalisé pour gérer les populations. Le « dispositif de guerre» que décrit Clausewitz est destiné à préparer un État à faire la guerre si elle se présente, à résoudre les conflits; or, le dispositif de sécurité de la gouvernementalisation, pour Foucault, n'a pas pour objectif de résoudre les conflits sociaux, mais bien d'en nier l'existence afin de les empêcher de survenir. Le parallèle serait donc plus exact si le dispositif de guerre d'un État, chez Clausewitz, avait pour but de dissuader les autres États de lui faire la guerre. Pour Foucault, le dispositif diplomatico-militaire de la gouvernementalité constitué au moment des traités de Westphalie recherche l'équilibre des rapports de force, soit par la guerre, soit par la possibilité de la guerre, ou soit par la menace de la guerre. Le caractère «libéral» de la biopolitique, évoquée dans le cours de 1979 (Naissance de la biopolitique), permet de dire que la menace de la guerre englobe et résume les deux autres solutions. En effet, un «gouvernement frugal» qui recherche l'efficacité au moindre coût placera l'essentiel de ses moyens dans la menace, donc dans la dissuasion. C'est d'ailleurs ce que les États occidentaux semblent avoir compris après le désastre des deux guerres mondiales du $\mathrm{XX}^{\mathrm{e}}$ siècle. De plus, la différence qu'elle conserve vis-à-vis du domaine de la science et des arts ferait de la guerre, si nous suivons Clausewitz, une activité que Foucault pourrait appeler «biopolitique».

37. K. von Clausewitz, De la guerre, livre II, p. 144-146. 
La différence essentielle réside en ce que la guerre n'est pas une activité de la volonté appliquée à une matière inerte, comme dans les arts mécaniques, ni à un objet vivant mais passif et soumis, tel que l'esprit humain et la sensibilité humaine dans les beaux-arts, mais à un objet qui vit et qui réagit ${ }^{38}$.

Le dispositif théorique de Clausewitz n'est pas de l'ordre de la géométrie qu'utilisent ses prédécesseurs, ni des disciplines de dressage qui considèrent le soldat comme soumis et docile, mais bien de l'ordre de la biologie, de la vie. L'objet de la guerre «vit et réagit» : il est vivant et résistant. Ce dispositif n'est donc pas non plus de l'ordre du «pouvoir-contrat» qui traite les objets vivants comme inertes, ni de celui de la "domination-répression» qui les suppose soumis, mais bien de l'ordre des "pouvoirs-résistances» qui caractériseront le monde de la biopolitique chez Foucault. Pour Clausewitz, «la politique est la matrice dans laquelle la guerre se développe ${ }^{39}$.

L'art de la guerre en général, et du commandement dans chaque espèce, peut exiger que les tendances et les intentions de la politique ne soient pas incompatibles avec ces moyens [la guerre], exigence non négligeable, assurément ${ }^{40}$.

La subordination de la guerre à la politique n'est pas une pure soumission, une pure obéissance. Clausewitz laisse entendre dès le livre I que les moyens de la guerre peuvent venir modifier la politique, que le commandement peut réagir aux intentions politiques, voire exiger qu'elles soient en cohérence avec les moyens militaires. Nous retrouvons ici aussi, dans les rapports politico-militaires, le schéma binaire «domination-résistance» ou "pouvoirsrésistances» caractéristique de la philosophie foucaldienne. Le livre VIII continue sur cette logique, mais en la justifiant d'après la méthode présentée dans la préface du traité.

Pour approfondir la contradiction qui existe dans la nature de la guerre par rapport aux autres intérêts humains, individuels ou sociaux, nous nous sommes tourné jusqu'à présent de tous les côtés, afin de ne négliger aucun de ces éléments antagoniques; comme cette contradiction est fondée sur l'homme lui-même, la raison philosophique ne peut la résoudre ${ }^{41}$.

Les contradictions observées dans l'expérience ne trouvent d'explication que lorsque l'on ramène la guerre réelle à un phénomène politique. De plus, même lorsque l'on envisage une guerre sans ces contradictions, sorte d'aspiration à se conformer au concept pur, celle-ci garde une contradiction

38. K. von Clausewitz, De la guerre, livre II, p. 144-146.

39. Ibid.

40. Ibid., livre I, p. 67.

41. Ibid., livre VIII, p. 703-704. 
en soi. Pour Clausewitz, même les guerres de Napoléon, qui sont la guerre absolue, fiction-réalité du concept de guerre, sont politiques.

Si la politique est grandiose et puissante, la guerre le sera aussi et pourra même atteindre les sommets où elle prend sa forme absolue. Dans cette conception, nous ne devons pas perdre de vue la forme absolue de la guerre et son image doit plutôt demeurer en permanence à l'arrière-plan. Seule cette conception de la guerre lui restitue son unité; seule elle nous permet de considérer toutes les guerres comme des choses d'un seul genre; seule elle donne le fondement précis et vrai et le point de vue qui permettrait d'élaborer et de juger de vastes plans ${ }^{42}$.

Le plan sur lequel se projette le concept de guerre, dans la forme absolue de la guerre, et sur lequel les guerres réelles se positionnent selon le niveau d'attraction des trois pôles (fig. 1), est un plan parcouru par la politique. Tout se passe comme si Clausewitz oscillait entre deux niveaux de réflexion philosophique: celui de l'ontologie, avec le concept de guerre, qui est un niveau apolitique, "indépendant»; et celui de la philosophie politique, qui analyse les rapports entre la politique et la guerre. Ce second niveau est celui de l'analytique de la guerre où Clausewitz envisage l'expérience issue des guerres réelles à la lumière de la forme absolue de la guerre. Ce projet est similaire à celui qu'aura Foucault sur le pouvoir : l'analytique du pouvoir observe l'exercice de différents pouvoirs - psychiatriques, judiciaires, disciplinaires, etc. - et les envisage à la lumière de la domination, cas limite dans lequel les résistances ne s'exercent plus, sorte de "pouvoir absolu». Mais, contrairement à Clausewitz, Foucault se refusera d'envisager un quelconque concept de pouvoir.

\section{Génie guerrier et peuple en armes}

Si nous projetions dans le monde des concepts, la forme absolue du pouvoir, la domination au sens fort, nous nous trouverions avec un concept qui exclut totalement la notion de réciprocité qui fait fonctionner le concept de guerre comme duel; nous aurions un concept dont l'essence même reposerait sur la non-réciprocité dans les relations. Nous aurions un anticoncept, en quelque sorte, de la guerre. Pourtant, c'est justement la guerre que Foucault tentera d'utiliser comme analyseur de pouvoir, comme outilcadre du plan dans lequel se déploie son analytique, c'est-à-dire le «plan d'immanence ${ }^{43}$ des rapports de pouvoir. Clausewitz, quant à lui, utilise

42. Ibid., p. 705 .

43. G. Deleuze, F. Guattari, Qu'est-ce que la philosophie? [1991], Paris, Minuit, 2005. 
la politique comme outil-cadre du plan d'immanence des guerres réelles. Nous l'avons vu, quand il s'agit de la guerre, la science et l'art, la rigueur et le talent, ne suffisent pas. Il faut décider, être capable de déterminer les fins de la guerre, si nécessaire de façon arbitraire. La plupart du temps, les éléments qui guident l'orientation des choix ne sont pas suffisants et la politique est dès lors indispensable. Les théories de la guerre montrent ainsi leurs limites.

Seule la partie analytique de ces tentatives de théorie constitue un progrès dans le domaine de la vérité; leur partie synthétique, leurs prescriptions et leurs règles sont tout à fait inutilisables. Elles visent des grandeurs certaines, alors qu'en guerre tout est incertain et que tous les calculs se font avec des grandeurs variables. Elles ne considèrent que des grandeurs matérielles, alors que l'acte de guerre est tout pénétré de forces et d'effets spirituels et moraux. Elles ne tiennent compte que de l'activité d'un seul camp, alors que la guerre repose sur l'action incessante que les deux camps exercent l'un sur l'autre ${ }^{44}$.

Trois aspects principaux de l'incertitude à la guerre empêchent de prescrire des règles d'action et imposent de savoir décider: les «frictions » ${ }^{45}$, les «forces morales» et la réciprocité. La théorie est donc seulement destinée à éduquer l'esprit du chef de guerre, non à l'accompagner à la bataille. Le chef de guerre, au-delà de sa rigueur - scientifique - et de son talent - artistique -, doit faire preuve du "génie qui s'élève au-dessus de la règle commune».

Ce que fait le génie, voilà la plus belle de toutes les règles, et ce que la théorie peut faire de mieux, c'est de montrer pourquoi il en est ainsi, et comment ${ }^{46}$.

Le génie guerrier de Clausewitz est un génie kantien ${ }^{47}$ : il est doué d'originalité car il donne ses règles à l'art; ses productions sont des modèles à suivre donc exemplaires; les idées de ses créations lui viennent naturellement. Clausewitz semble ainsi rattacher la pratique de la guerre au domaine des beaux-arts, plutôt qu'à celui de la politique. Or Hannah Arendt ${ }^{48}$ a montré que la troisième critique de Kant, la Critique de la faculté de juger qui porte sur le beau - où se retrouve la notion de talent - et le sublime - où se

44. K. von Clausewitz, De la guerre, livre II, p. 128-129.

45. Le concept de friction, introduit par Clausewitz, recouvre un domaine plus large que le hasard; ce peut être le brouillard ou la pluie; il est «ce qui rend difficile tout ce qui paraît facile» : ibid., livre I, p. 111.

46. Ibid., livre II, p. 128-129.

47. E. Kant, Critique de la faculté de juger [1790], A. Renaut (trad.), Paris, Flammarion, 2000, $\$ 46$.

48. H. Arendt, Juger. Sur la philosophie politique de Kant, Paris, Seuil, 2003. 
retrouve la notion de génie - était celle qui développait la pensée politique de Kant. Nous pourrions alors avancer que le chef de guerre doit faire preuve, suivant l'idée du génie guerrier, de «sens politique» : savoir décider dans l'action, sans se conformer aux règles établies mais sans pour autant paraître arbitraire, de sorte que la postérité jugera la décision exemplaire et que l'action deviendra un «canon» de l'art de la guerre. Il n'est pas étonnant, dans ses conditions, que Napoléon, homme politique et chef de guerre, ait été le «Dieu de la guerre» pour Clausewitz. La subordination de la guerre au politique rappelle que la guerre ne peut pas se faire sans sens politique.

L'inspiration kantienne est également visible dans l'appellation des trois pôles d'attraction que sont l'impulsion naturelle, l'entendement pur et la libre activité de l'âme. Ceux-ci rappellent, de près ou de loin, les trois principales facultés humaines selon Kant ${ }^{49}$ : la sensibilité qui est ce par quoi un objet nous est donné, l'entendement qui est la faculté des concepts, et la raison qui est celle des principes. L'impulsion naturelle, comme la sensibilité chez Kant, a une position privilégiée dans la théorie clausewitzienne: elle est ce par quoi l'objet "guerre réelle» nous est donné. Sans elle, la partie analytique de la théorie de la guerre serait sans objet, du moins si l'on ne veut pas se contenter de la facilité des certitudes. Pour Clausewitz, la guerre moderne met en évidence la primauté des forces morales. Elle est celle des passions du peuple, celle de la nation en armes de Napoléon, mais aussi celle de la guérilla espagnole contre les forces d'occupation napoléoniennes.

Finalement, ces événements ont tous montré quel facteur immense le cœur et le sentiment d'une nation peuvent représenter dans le produit des forces de l'État, de la guerre et du combat, et maintenant que les gouvernements ont appris à connaître tous ces moyens additionnels, il ne faut pas s'attendre à ce qu'ils les laissent inactifs dans les guerres futures, si le danger menace leur existence même, ou si quelque ambition fiévreuse les y entraine ${ }^{50}$.

Les guerres réelles, au XVIII ${ }^{\mathrm{e}}$ siècle, oscillaient finalement entre les deux pôles de l'entendement pur, qu'incarnait le gouvernement politique, et de la libre activité de l'âme, qu'incarnaient le chef de guerre et son armée. Avec la Révolution et Napoléon, le pôle de l'impulsion naturelle s'est mis à attirer la guerre vers lui et l'a ainsi rapprochée de la guerre absolue, sorte de barycentre entre les trois pôles.

49. E. Kant, Critique de la raison pure [1781], A. Renaut (trad.), Paris, Flammarion, 2006.

50. K. von Clausewitz, De la guerre, livre III, p. 232. 
Clausewitz se place au tournant de ce que Foucault appellera la biopolitique: la partie analytique de sa théorie nous fait encore osciller entre la «raison d'État» foucaldienne, qu'assoient les réflexions sur les guerres réelles et leur limitation par la politique, et la nouvelle rationalité de la biopolitique, qui met au centre de ses préoccupations ce qui est vivant et réagit, mais qui, œuvrant à l'enrichissement et donc à l'amélioration des conditions de vie de tous, libère aussi les forces résistantes des peuples. Clausewitz a annoncé l'illimitation des guerres futures: les guerres absolues qu'a menées Napoléon, les guerres des peuples en armes comme celle d'Espagne, lui ont montré que le pouvoir politique de l'État ne pouvait pas, ou ne voulait pas, forcément maitriser les forces morales ainsi libérées. Foucault nous montre que le congrès de Vienne a permis de rendre inaperçu le passage de la raison diplomatico-politique à la rationalité de la concurrence et des intérêts particuliers. Officiellement, l'équilibre européen était préservé; insidieusement, la course pour les aspirations économiques ou nationales avait commencé. Le XIX ${ }^{\mathrm{e}}$ et le $\mathrm{XX}^{\mathrm{e}}$ siècles se sont ensuite embrasés. La polémique née des lectures à tendance libérale de Clausewitz ne fait que confirmer cette thèse de Foucault sur la dialectisation du discours sur la guerre. Ces lectures font de la violence déchaînée des États une irréalité politique $^{51}$. Il semble pourtant bien que ce ne soit là qu'un discours qui se veut performatif. Seules les stratégies de dissuasion mises en œuvre après la Seconde Guerre mondiale paraissent avoir atteint la mise entre parenthèses des guerres interétatiques.

La lecture de De la guerre nous permet également de confronter deux analytiques: celle du pouvoir chez Foucault et celle de la guerre chez Clausewitz. Ce dernier alterne néanmoins cette méthode de pensée, contrairement à Foucault, avec une réflexion d'un autre niveau philosophique sur le concept de guerre lui-même. D'ailleurs, l'imbrication des niveaux de réflexion - même si clairement annoncée -, et donc de leur objet respectif, la guerre réelle et le concept de guerre, a donné lieu à des interprétations erronées ${ }^{52}$. L'ouvrage De la guerre ne peut être compris si l'on ne fait pas la distinction entre le niveau de réflexion de l'ontologie de la guerre et celui de la philosophie politique dans lequel se développe une analytique de la guerre. Nous avons proposé un parallèle, ou plutôt une lecture en miroir, entre cette analytique et celle de Foucault. Dans le plan d'immanence du

51. De la même façon, la guerre illimitée à l'intérieur de la société, insidieuse, cachée, est un autre oubli volontaire de la pensée politique. Cet oubli de la guerre civile, ou plus précisément de la stasis, depuis l'Antiquité romaine est montré par N. Grangé, Oublier la guerre civile? "Stasis", chronique d'une disparition, Paris, J. Vrin - Éditions de l'EHESS, 2015.

52. B. H. Liddell Hart (Stratégie [1954], L. Poirier (éd. et trad.), Paris, Perrin, 2007, p. 545-546) accuse Clausewitz d'être à l'origine de la guerre totale moderne. 
pouvoir foucaldien, l'outil d'analyse «guerre» introduirait la réciprocité qui fonde la possibilité des rapports de force et qui permet d'appréhender le pouvoir, non dans sa forme stérile de pouvoir absolu, de domination pure, mais selon un schéma "pouvoirs-résistances». De façon symétrique, dans le plan d'immanence de la guerre clausewitzien, l'outil d'analyse "politique " introduirait l'antithèse de la réciprocité, qui enraye la montée aux extrêmes et qui admet l'asymétrie des rapports de force et les «frictions» de la guerre.

L'outil «guerre» permet à Foucault de penser le pouvoir dans l'immanence des rapports de force sans faire appel à la transcendance d'un pouvoir imaginaire du prince ou de l'autorité spirituelle et symbolique du roi, de la même façon que l'outil "politique" permet à Clausewitz de se dégager des axiomes de réciprocité pure et de penser la guerre comme freinée par la politique. Finalement, il semble que le lien "guerre-politique» ne puisse pas être défait et que les deux objets «guerre» et «politique» évoluent dans un rapport indissociable d'inversion l'un par rapport à l'autre. La force de la formule clausewitzienne semble être alors sa capacité à susciter le questionnement autour de son renversement: politiquement, il est souhaitable que la guerre soit la continuation de la politique par d'autres moyens; philosophiquement, il est nécessaire de ne pas occulter le fait que la politique puisse être une guerre continuée. «Guerre» et «politique» seraient comme l'avers et le revers d'une même pièce qui tourne sur elle-même, lancée dans le flot de l'histoire. 
